\title{
Factores relacionados al nivel de práctica del autoexamen de mamas, Centro de Salud Anta, Carhuaz, 2015
}

\author{
Factors related to the level of practice of breast self-examination, Anta \\ Health Center, Carhuaz, 2015
}

\author{
Augusto Olaza Maguiña ${ }^{1}$, Yuliana de la Cruz Ramírez ${ }^{1}$ Y \\ Víctor Olaza Maguiña ${ }^{2}$
}

\section{RESUMEN}

Determina los factores relacionados al nivel de práctica del autoexamen de mamas en mujeres que acuden al Centro de Salud Anta, Carhuaz, 2015. Se realizó una investigación prospectiva, observacional y correlacional en una muestra de 271 mujeres. Se usó un cuestionario y una guía de observación. La información se procesó mediante el programa SPSS V20.0, utilizando la prueba Chi cuadrado. Los resultados evidenciaron que la mayoría de mujeres tenía un bajo nivel de práctica del autoexamen de mamas (51,7\%). Aquí se obtiene entre 20 y 35 años el (24,7\%); convivientes (38,1\%); con estudios del nivel secundario (28,4\%); procedentes del área rural (41,3\%); amas de casa $(43,5 \%)$ y sin ingreso económico propio (47,6\%). Se concluye que los factores demográficos y sociales están relacionados con nivel de práctica del autoexamen de mamas en mujeres que acuden al Centro de Salud Anta de Carhuaz, con excepción del estado civil.

Palabras clave: factor; práctica; autoexamen; mama.

\section{ABSTRACT}

This research determines the factors related to the level of practice of breast selfexamination in women who come to the Anta Health Center, Carhuaz, 2015, for which a prospective, observational and correlational research was conducted in a sample of

1 Universidad Nacional «Santiago Antúnez de Mayolo». Huaraz, Perú.

2 Dirección Regional de Salud Ancash. Huaraz, Perú. 
271 women. A questionnaire and an observation guide were used. The information was processed by means of SPSS V20.0 software, using Chi Square test. The results showed that the majority of women had a low level of practice of breast self-examination (51,7\%), showing between 20 and 35 years of age (24,7\%), cohabiting relationship $(38,1 \%)$, with studies of high school $(28,4 \%)$, from the rural area $(41,3 \%)$, housewives $(43,5 \%)$ and without own income $(47,6 \%)$. It was concluded that demographic and social factors are related to the level of practice of breast self-examination in women who come to the Anta Health Center of Carhuaz, except marital status.

Keywords: factor; practice; self-examination; breast.

\section{ICHIKLLACHAW}

Kay musyapakuyqa Anta hampikuna wayichaw, Qarwashpa markan, warmikunapa chichinkuna kikinkuna rikapayanqanpaqmi riqitsimantsik, kayta rurakashqa 2015 watachaw. Kayqa pruspiktiba, qawapakuy, kurilasiyunal musyapakuymi rurakashqa, 271 warmikunachaw. Tsaypaq tapukuykunatawan tapukuy rikaanatam wanakashqa. Kay ashipakuy willakuyqa SPSS V20.0 prugrama nishqanwanmi rurakashqa, Chi kuwadradu chanintsay nishqanwan. Kayqa hitamushqa lutaypa warmikunapa chichinkuna kikinkuna rikapaayanqanta uchukllam rurayan, kay (51,7\%) llamanmi chan. Kaychaw hurqaqashqa 20piqwan 35 watayuq (24,7\%); mahankunawan yachaqkuna (38\%); ishkaq kaq musyapakuypa (28,4\%); tuna markapiq shamuqkuna $(41,3 \%)$; warmikuna wayichaw yanapakuqkuna (43,5\%) niykur mana qillay chaskiqkuna (47,6\%). Ushanapaq nintsik, llaqtachaw yachaqkuna warmikunapa chichinkuna kikinkuna rikapakuqkuna tinkukan Anta hampikuna wayita aywaqkunachaw-Qarwash marka.

Pushaq shimikuna: huwaktur; ruraynin; kikinkuna rikapayanqan; chichi.

\section{INTRODUCCIÓN}

La presente investigación aborda el nivel de práctica del autoexamen de mamas en la población de mujeres que se atiende en el Centro de Salud Anta, establecimiento de salud de categoría I-4 de nivel de atención, ubicado en el distrito del mismo nombre, provincia de Carhuaz, Áncash. La población que se atiende en dicho establecimiento procede en un 68\% del área rural, con un nivel educativo promedio de secundaria incompleta, hablantes bilingües quechua-español en más del 54\% y un promedio de ingreso económico mensual apenas superior al sueldo mínimo vital (Dirección Regional de Salud Áncash, 2015).

El autoexamen de mamas es una técnica que consiste en la propia observación y palpación que la mujer hace de sus mamas. Sirve para poder detectar la aparición de alguna 
alteración en la forma o el tamaño normal (Ministerio de Salud, 2004) La mamografía es la herramienta diagnóstica más utilizada en los programas de tamizaje y diagnóstico del cáncer de mama (World Health Organization, 2015); sin embargo, el autoexamen de mamas es de bajo costo y no invasivo; además, tiene la ventaja de ser llevado a cabo por la propia mujer. Su conocimiento puede llevar a un diagnóstico temprano del cáncer de mama, sobre todo en países con escasos recursos económicos que no cuentan con infraestructura suficiente para el tamizaje poblacional con mamografía (Ibíd.).

Existen trabajos de investigación sobre el nivel de práctica del autoexamen de mamas, sobre todo a nivel internacional, en los cuales la mayoría de las investigaciones dan como resultado que las mujeres tienen un nivel de práctica bajo. Al respecto, Arroyo et al. (2008), encontró que la mayoría de mujeres del área rural boliviana (58\%), no identifica el momento, frecuencia y tiempo de duración del autoexamen de mamas. Respecto a la realización del autoexamen de mamas en el Perú, pocos estudios han determinado su frecuencia. Un estudio en Sullana, en el norte del Perú, reportó que 57\% de las mujeres estudiadas, nunca se realizó un autoexamen de mamas, mientras que el 56\% refirió conocer la utilidad del mismo (Villacrés y Alarcón, 2002). Otro estudio, efectuado en dos distritos de la costa peruana, encontró una frecuencia del autoexamen de mamas del 15\%. Al consultar a las participantes del por qué no se realizaban el autoexamen de mamas, éstas respondieron desconocer del tema (Alarcón, Villacrés y Cárdenas, 2005).

Debido a la importancia del tema, y en ausencia de estudios similares a nivel local, se realizó la presente investigación con el objetivo de determinar los factores relacionados al nivel de práctica del autoexamen de mamas en mujeres que acuden al Centro de Salud Anta de Carhuaz durante el año 2015. Los objetivos específicos fueron: Identificar el nivel de práctica del autoexamen de mamas; determinar los factores demográficos relacionados al nivel de práctica del autoexamen de mamas; y, precisar los factores sociales que se relacionan al nivel de práctica del autoexamen de mamas.

\section{MATERIALES Y MÉTODOS}

Se llevó a cabo un estudio prospectivo, transversal y correlacional, con un diseño de tipo no experimental, transeccional o transversal correlacional (Hernández, Fernández y Baptista, 2010; Canales, Alvarado y Pineda, 2006). La población estuvo conformada por 1734 mujeres con una edad mayor o igual a 15 años, quienes fueron atendidas durante el año 2015 en el servicio de Gineco-Obstetricia del Centro de Salud Anta. Se usó el muestreo probabilístico, para lo cual se determinó el tamaño de la muestra, mediante la aplicación de la fórmula respectiva (Hopkins, 2008), y se obtuvo una muestra de 271 mujeres. 
La información se obtuvo usando como instrumentos un cuestionario y una guía de observación, previa autorización del consentimiento informado. La validez de los instrumentos se realizó mediante juicio de expertos, cuya prueba de concordancia de Kendall, demostró su validez con un nivel de significancia del 0,0002 y 0,001, respectivamente. Asimismo, luego de la aplicación de una prueba piloto, se procedió al cálculo de la confiabilidad de los instrumentos mediante el Coeficiente alfa de Cronbach, y se obtuvo como resultado instrumentos de investigación confiables $(0,854$ y 0,804 , respectivamente).

Para el procesamiento de la información se usó el Paquete Estadístico para las Ciencias Sociales V20.0. (IBM Corporation, 2012), utilizándose como instrumento de análisis las tablas y gráficos de frecuencia, así como la prueba Chi cuadrado, con un nivel de significancia del 5\% ( $<0$,05) (Freund, 2009).

\section{RESULTADOS}

En la tabla 1 se evidencia que el 51,7\% del total de mujeres consideradas en el presente estudio (271), tiene un bajo nivel de práctica del autoexamen de mama, según los indicadores establecidos en la guía de observación aplicada.

Tabla 1. Nivel de práctica del autoexamen de mamas

\begin{tabular}{ccc}
\hline Nivel & $\mathrm{N}^{\circ}$ & $\%$ \\
\hline Alto (15-21 puntos) & 95 & $35,1 \%$ \\
Medio (8 -14 puntos) & 36 & $13,2 \%$ \\
Bajo ( $\leq 7$ puntos $)$ & 140 & $51,7 \%$ \\
Total & 271 & $100 \%$ \\
\hline
\end{tabular}

En la tabla 2, se observa que el mayor porcentaje de bajo nivel de práctica del autoexamen de mamas, correspondió a mujeres con una edad comprendida entre los 20 y 35 años $(24,7 \%)$, convivientes $(38,1 \%)$ y con estudios del nivel secundario $(28,4 \%)$, evidenciándose que, a excepción del estado civil $(\mathrm{p}>0,05)$, el resto de variables como la edad y el grado de instrucción, presentaron una relación estadísticamente significativa con el nivel de práctica del autoexamen de mamas $(\mathrm{p}<0,05)$. 
Tabla 2. Factores demográficos relacionados al nivel de práctica del autoexamen de mamas

\begin{tabular}{|c|c|c|c|c|c|c|c|c|c|}
\hline \multirow{3}{*}{ Factores demográficos } & \multicolumn{6}{|c|}{ Nivel de práctica } & \multirow{2}{*}{\multicolumn{2}{|c|}{ Total }} & \multirow{3}{*}{$\begin{array}{l}\text { Resultado de } \\
\text { la prueba Chi } \\
\text { cuadrado }\end{array}$} \\
\hline & \multicolumn{2}{|c|}{ Alto } & \multicolumn{2}{|c|}{ Medio } & \multicolumn{2}{|c|}{ Bajo } & & & \\
\hline & $\mathrm{N}^{\circ}$ & $\%$ & $\mathrm{~N}^{\circ}$ & $\%$ & $\mathrm{~N}^{\circ}$ & $\%$ & $\mathrm{~N}^{\circ}$ & $\%$ & \\
\hline Edad & & & & & & & & & \\
\hline $15-19$ años & 10 & 3,7 & 2 & 0,7 & 24 & 8,9 & 36 & 13,3 & \\
\hline $20-35$ años & 56 & 20,7 & 18 & 6,6 & 67 & 24,7 & 141 & 52 & $X^{2}=15,555$ \\
\hline $36-49$ años & 19 & 7 & 16 & 5,9 & 41 & 15,1 & 76 & 28 & $p^{c}=0,016$ \\
\hline$\geq 50$ años & 10 & 3,7 & 0 & 0 & 8 & 3 & 18 & 6,7 & \\
\hline Total & 95 & 35,1 & 36 & 13,2 & 140 & 51,7 & 271 & 100 & \\
\hline Estado civil & & & & & & & & & \\
\hline Soltera & 10 & 3,7 & 4 & 1,5 & 15 & 5,5 & 29 & 10,7 & \\
\hline Casada & 15 & 5,5 & 6 & 2,3 & 21 & 7,7 & 42 & 15,5 & $\mathrm{X}^{2}=0,473$ \\
\hline Conviviente & 69 & 25,5 & 26 & 9,4 & 103 & 38,1 & 198 & 73 & $\mathrm{p}=0,998$ \\
\hline Viuda & 1 & 0,4 & 0 & 0 & 1 & 0,4 & 2 & 0,8 & \\
\hline Total & 95 & 35,1 & 36 & 13,2 & 140 & 51,7 & 271 & 100 & \\
\hline Grado de instrucción & & & & & & & & & \\
\hline Analfabeta & 0 & 0 & 2 & 0,7 & 10 & 3,7 & 12 & 4,4 & $\mathrm{X}_{\mathrm{c}}^{2}=77,394$ \\
\hline Primaria & 1 & 0,4 & 6 & 2,2 & 52 & 19,2 & 59 & 21,8 & $\mathrm{p}^{\mathrm{c}}=0,000$ \\
\hline Secundaria & 80 & 29,5 & 18 & 6,7 & 77 & 28,4 & 175 & 64,6 & \\
\hline Superior & 14 & 5,2 & 10 & 3,6 & 1 & 0,4 & 25 & 9,2 & \\
\hline Total & 95 & 35,1 & 36 & 13,2 & 140 & 51,7 & 271 & 100 & \\
\hline
\end{tabular}

En la tabla 3 se evidencia que la mayoría de mujeres con bajo nivel de práctica del autoexamen de mamas, fue procedente de la zona rural (41,3\%), amas de casa $(43,5 \%)$ y sin un ingreso económico propio $(47,6 \%$ ). Asimismo, se encontró una relación estadísticamente significativa de la procedencia, ocupación e ingreso económico con el nivel de práctica del autoexamen de mamas $(\mathrm{p}<0,05)$.

Tabla 3. Factores sociales relacionados al nivel de práctica del autoexamen de mamas

\begin{tabular}{|c|c|c|c|c|c|c|c|c|c|}
\hline \multirow{3}{*}{ Factores sociales } & \multicolumn{6}{|c|}{ Nivel de práctica } & \multirow{2}{*}{\multicolumn{2}{|c|}{ Total }} & \multirow{3}{*}{$\begin{array}{l}\text { Resultado de } \\
\text { Chi cuadrado }\end{array}$} \\
\hline & \multicolumn{2}{|c|}{ Alto } & \multicolumn{2}{|c|}{ Medio } & \multicolumn{2}{|c|}{ Bajo } & & & \\
\hline & $\mathrm{N}^{\circ}$ & $\%$ & $\mathrm{~N}^{\circ}$ & $\%$ & $\mathrm{~N}^{\circ}$ & $\%$ & $\mathrm{~N}^{\circ}$ & $\%$ & \\
\hline Procedencia & & & & & & & & & \\
\hline $\begin{array}{c}\text { Zona urbana ( } \geq 100 \text { viviendas } \\
\text { agrupadas contiguamente) }\end{array}$ & 51 & 18,8 & 6 & 2,2 & 28 & 10,3 & 85 & 31,4 & $\mathrm{X}_{\mathrm{c}}^{2}=33,996$ \\
\hline $\begin{array}{l}\text { Zona rural (<100 viviendas } \\
\text { agrupadas contiguamente) }\end{array}$ & 44 & 16,2 & 30 & 11,1 & 112 & 41,3 & 186 & 68,6 & $\mathrm{p}=0,000$ \\
\hline Total & 95 & 35,1 & 36 & 13,2 & 140 & 51,7 & 271 & 100 & \\
\hline $\begin{array}{c}\text { Ocupación } \\
\text { Ama de casa } \\
\text { Estudiante } \\
\text { Comerciante } \\
\text { Empleada del sector público } \\
\text { Total }\end{array}$ & $\begin{array}{c}36 \\
5 \\
51 \\
3 \\
95\end{array}$ & $\begin{array}{c}13,4 \\
1,8 \\
18,8 \\
1,1 \\
35,1\end{array}$ & $\begin{array}{c}15 \\
6 \\
12 \\
3 \\
36\end{array}$ & $\begin{array}{c}5,5 \\
2,2 \\
4,4 \\
1,1 \\
13,2\end{array}$ & $\begin{array}{c}118 \\
11 \\
9 \\
2 \\
140\end{array}$ & $\begin{array}{c}43,5 \\
4,1 \\
3,4 \\
0,7 \\
51,7\end{array}$ & $\begin{array}{c}169 \\
22 \\
72 \\
8 \\
271\end{array}$ & $\begin{array}{c}62,4 \\
8,1 \\
26,6 \\
2,9 \\
100\end{array}$ & $\begin{array}{c}\mathrm{X}^{2}=79,519 \\
\mathrm{p}^{\mathrm{c}}=0,000\end{array}$ \\
\hline $\begin{array}{c}\text { Ingreso económico } \\
\text { Con ingreso propio } \\
\text { Sin ingreso propio } \\
\text { Total }\end{array}$ & $\begin{array}{l}54 \\
41 \\
95\end{array}$ & $\begin{array}{l}19,9 \\
15,2 \\
35,1\end{array}$ & $\begin{array}{l}15 \\
21 \\
36\end{array}$ & $\begin{array}{r}5,5 \\
7,7 \\
13,2\end{array}$ & $\begin{array}{c}11 \\
129 \\
140\end{array}$ & $\begin{array}{l}4,1 \\
47,6 \\
51,7\end{array}$ & $\begin{array}{c}80 \\
191 \\
271\end{array}$ & $\begin{array}{l}29,5 \\
70,5 \\
100\end{array}$ & $\begin{array}{c}\mathrm{X}^{2}=68,216 \\
\mathrm{p}^{\mathrm{c}}=0,000\end{array}$ \\
\hline
\end{tabular}




\section{DISCUSIÓN}

Los resultados de la presente investigación con respecto al primer objetivo específico, orientado a identificar el nivel de práctica del autoexamen de mamas en mujeres que acuden al Centro de Salud Anta de Carhuaz, fueron dados a conocer en la tabla 1, en la cual se evidenció que el 51,7\% del total de mujeres consideradas en el presente estudio (271), tiene un bajo nivel de práctica del autoexamen de mama; hallazgo coincidente con el estudio realizado por los autores Cerquera et al. (2010), quienes encontraron que solo un 37\% de mujeres de la ciudad de Neiva en Colombia, realizaban el autoexamen de sus senos, lo cual, tal y como lo mencionan Del Toro, Peinado y Díaz (2014) en su investigación realizada en el mismo país de Colombia, se debe al desconocimiento que existe con respecto a esta práctica preventiva $(67,2 \%)$.

Por su parte, Martínez et al. (2015), en su estudio de tipo descriptivo, con respecto a las barreras asociadas a la realización del autoexamen de seno en mujeres de 18 a 50 años de edad, encontraron un porcentaje mayor de bajo nivel de práctica, ya que el $72 \%$ de las mujeres encuestadas, aceptó no haberse practicado el autoexamen de mama en el último mes, y el 45\% en los últimos seis meses, motivo por el cual enfatizó en la necesidad de ejecutar programas de intervención educativa acerca de la importancia del diagnóstico temprano del cáncer de mama; mientras que Virella et al. (2009) en Cuba, determinaron que aunque es un tema importante para la población femenina, solo el $8,3 \%$ de ellas lo ha realizado alguna vez en su vida.

La información mencionada anteriormente sobre estudios desarrollados en otros países, es coincidente con el estudio llevado a cabo en el Perú por Romaní, Gutiérrez y Ramos (2011), quienes llevaron a cabo un estudio de análisis de fuentes secundarias en base a la Encuesta Demográfica y de Salud Familiar (ENDES) del año 2008, en el cual se investigó la dinámica demográfica y el estado de salud materno infantil, incluyendo la pregunta acerca de la realización del autoexamen de mama, cuya prevalencia alcanzó al 34,6\% de las mujeres peruanas de 20 a 49 años, lo cual significaría un 65,4\% de promedio nacional en cuanto a la no aplicación de dicha medida preventiva por parte de las mujeres peruanas, cifra que es superior a la encontrada en el presente estudio, la cual es más cercana a la dada a conocer en el estudio de Callata y Quispe (2011), quienes identificaron que solo un 55\% de mujeres que acuden al Centro de Salud Leonor Saavedra en la ciudad de Lima, alguna vez ha realizado la autoexploración mamaria y un $45 \%$ nunca lo ha hecho. Asimismo, el 55\% de mujeres que lo ha realizado, lo hizo con una frecuencia mínima de tres meses, solo un 18,2\% está lo más cercano a un mes en frecuencia, mientras que ninguna mujer cumplió con la frecuencia en que se debe realizar el autoexamen de mamas; realidad muy similar a la reportada por Sáenz y Sánchez (2011) en el Hospital Nacional Daniel Alcides Carrión del Callao y Pacohuanaco (2012) en Tacna. 
Resultados opuestos a los mencionados, fueron obtenidos por Yépez et al. (2012), quienes encontraron que el $82,8 \%$ de las mujeres que acude al Instituto Mexicano del Seguro Social, sabe qué es la autoexploración mamaria, observándose que el 73,7\% de las mujeres se realiza dicha autoexploración; hallazgos que los autores, asumen que se debe a las diferencias en las características sociodemográficas de su población.

En las tablas 2 y 3, se pone de manifiesto las respuestas al tercer y cuarto objetivo específico del presente estudio, orientados a determinar los factores demográficos y sociales que se relacionan con nivel de práctica del autoexamen de mamas, habiéndose encontrado que, a excepción del estado civil, el resto de factores como la edad, grado de instrucción, procedencia, ocupación e ingreso económico, sí tienen una relación estadísticamente significativa con el nivel de práctica del autoexamen de mamas; conclusión similar a la dada a conocer por los autores Martínez et al. (2015), quienes encontraron que la edad de las mujeres correspondientes al grupo etario de 30 a 39 años, se asoció como una barrera para el autoexamen de mamas; entretanto que Ospina et al. (2011), dieron a conocer que los factores que se encontraron asociados con la no realización del autoexamen mamario en la ciudad de Tunja, en Colombia, fueron el no contar con apoyo familiar $(\mathrm{OR}=3,18)$, no haber terminado la secundaria $(\mathrm{OR}=1,94)$ y el no trabajar $(\mathrm{OR}=1,47)$; los cuales son factores susceptibles de intervención educativa que podrían mejorar sustancialmente con programas adecuados de prevención y promoción. Manrique et al. (2012), por su parte, concluyeron también en Tunja-Colombia, como uno de los principales factores asociados con la realización adecuada del autoexamen de mamas, a la escolaridad secundaria o más, siendo preciso el reforzar la educación en salud y motivar a las mujeres para que se realicen oportuna y correctamente esta práctica, situación que debe ser considerada por los organismos de salud.

A nivel del Perú, la investigación de Romaní, Gutiérrez y Ramos (2011), concluyó que los factores sociodemográficos asociados a la realización del autoexamen de mamas, fueron el tener una edad entre 30 a 49 años y el poseer índice de riqueza medio, rico, o muy rico; mientras que el estudio de Callata y Quispe (2011), encontraron que los factores demográficos y socioeconómicos como la escolaridad y el manejar un ingreso familiar menor de 500 soles mensuales, pueden influenciar en el grado de conocimiento de las mujeres sobre el autoexamen de mamas, y por consiguiente en su realización.

Finalmente, la reflexión epistemológica sobre los resultados antes mencionados, permite señalar que el abordaje del nivel de práctica del autoexamen de mamas en la población de mujeres que se atienden en el Centro de Salud Anta de Carhuaz, solo se limita a una concepción médico-asistencialista, dejándose de lado factores de tipo demográfico y sociales, los cuales también son importantes cuando se habla sobre temas de prevención y promoción de la salud, los cuales por sus características de orden cultural y social, necesitan de la aplicación de un enfoque con perspectiva de género, basado en la atención inter y multidisciplinaria, según la realidad en la que se desenvuelven las mujeres. 


\section{CONCLUSIONES}

Los factores demográficos y sociales tienen una relación estadísticamente significativa con el nivel de práctica del autoexamen de mamas en mujeres que acuden al Centro de Salud Anta de Carhuaz, con excepción del estado civil.

La mayoría de mujeres que acuden al Centro de Salud Anta de Carhuaz tienen un bajo nivel de práctica del autoexamen de mamas, seguido de aquellas con un nivel de práctica alto y medio.

Los factores demográficos que guardan una relación estadísticamente significativa con el nivel de práctica del autoexamen de mamas, son la edad y el grado de instrucción; no sucede lo mismo con el estado civil.

La procedencia, ocupación e ingreso económico, se constituyen en factores sociales que tienen una relación estadísticamente significativa con el nivel de práctica del autoexamen de mamas.

\section{AGRADECIMIENTOS}

Al personal directivo y asistencial del Centro de Salud Anta de Carhuaz por su colaboración durante el desarrollo del presente estudio.

\section{REFERENCIAS BIBLIOGRÁFICAS}

Alarcón, Ana; Villacrés, Karina y Cárdenas, Raúl. 2005. «Perceptions of breast and cervix cancer prevention in two populations of women in Peru: Impact of knowledge and access to health service».Clin Oncol ASCO AnnualMeeting Proceedings, Vol. 23, N 16S. Alexandria: American Society of Clinical Oncology. 3653-3660.

Arroyo, Dolly et al. 2008. Conocimientos y práctica que tienen las mujeres en edad fértil sobre el autoexamen de mama en el Barrio de Juan Pablo II de la provincia Cercado de la ciudad de Tarija gestión 2008. Tarija: Universidad Autónoma Juan Misael Saracho. <http:// slidegur.com/doc/1619396/descargar-en-multmiedia---facultad-de-cienciasde-la-salud $>$ [Consulta: 28-10-2015].

Callata, Yolanda y Quispe, Nélida. 2011. Factores relacionados con los conocimientos y las actitudes hacia el autoexamen de mamas en mujeres en edad fértil que acuden a la consulta externa del C. S. Leonor Saavedra - año 2011. Tesis de Licenciatura, Universidad Nacional Mayor de San Marcos.

Canales, Francisca; Alvarado, Eva y Pineda, Elia. 2006. Metodología de la investigación. Manual para el desarrollo de personal de salud. México D. F.: Limusa.

Cerquera, Johan et al. 2010. «Autoexamen de seno, como prevención en las adolescentes de colegios públicos y privados de la ciudad de Neiva en el 2009». Revista Facultad de Salud-RFS, Vol. 2, N². Neiva: Universidad Surcolombiana. 49-54. 
Del Toro, Moraima; Peinado, Lindsay y Díaz, Anderson. 2014. «Conocimientos y prácticas sobre el autoexamen mamario en mujeres de Cartagena de Indias (Bol. - Col)». Ciencia e Innovación en Salud, Vol. 2, N 1. Barranquilla: Universidad Simón Bolívar. 27-32.

Dirección Regional de Salud Áncash. 2015. Reporte estadístico de producción. Huaraz: DIRESA.

Freund, Jhon. 2009. Estadística elemental. México D. F.: Prentice-Hall Hispanoamericana.

Hernández, Roberto; Fernández, Carlos y Baptista, Pilar. 2010. Metodología de la investigación. 5ta. ed. México D. F.: McGraw-Hill.

Hopkins, Billy. 2008. Estadística básica para las ciencias sociales y del comportamiento. México D. F.: Prentice-Hall Hispanoamericana.

IBM Corporation. 2012. Statistics program for Social Science V20.0 [CD-ROM]. New York: IBM Corporation.

Manrique et al. 2012. «Factores asociados a la práctica correcta del autoexamen de mama en mujeres de Tunja (Colombia)». Revista Investigación y Educación en Enfermería, Vol. 30, N 1. Medellín: Universidad de Antioquia. 18-27.

Martínez, Javier et al. 2015. «Barreras asociadas a la realización del autoexamen de seno en mujeres de 18 a 50 años de edad: un estudio descriptivo». Nutrición hospitalaria, Vol. 32, N 4. Madrid: Grupo Arán. 1664-1669.

Ministerio de Salud. 2004. Guías nacionales de atención integral de la salud sexualy reproductiva. Lima: MINSA.

Ospina et al. 2011. «La no realización del autoexamen de mama en Tunja, Colombia». Revista Enfermería Global, No 23, Murcia, Universidad de Murcia. < http:/ / revistas.um.es/eglobal/article/view/119211/122631> [Consulta: 31-10-2015].

Pacohuanaco, Maritza. 2012. Conocimiento, actitudy su relación con la práctica del autoexamen de mama en adolescentes del nivel secundario de la Institución Educativa Mariscal Cáceres Tacna-2012. Tesis de Licenciatura, Universidad Nacional Jorge Basadre Grohmann.

Romaní, Franco; Gutiérrez, César y Ramos-Castillo, José. 2011. «Autoexamen de mama en mujeres peruanas: prevalencia y factores sociodemográficos asociados. Análisis de la Encuesta Demográfica de Salud Familiar (ENDES)». Anales de la Facultad de Medicina, Vol. 72, N 1. Lima: Universidad Nacional Mayor de San Marcos. 23-31.

Sáenz, Andrea y Sánchez, Ángela. 2011. Conocimientos, actitudes y prácticas del autoexamen de mama en mujeres de 15 - 45 años que acuden a los consultorios de Planificación Familiar y Papanicolaou del Hospital Nacional Daniel Alcides Carrión, periodo agosto - diciembre del 2010. Tesis de Licenciatura, Universidad Nacional Mayor de San Marcos. 
Villacrés, Karina y Alarcón, Ana. 2002. «Estudio piloto sobre actitudes preventivas para cáncer de mama en mujeres de Sullana». Revista Médica Herediana, No 13. Lima: Universidad Peruana Cayetano Heredia. 131-134.

Virella, María et al. 2009. «Resultados del conocimiento sobre factores de riesgo del cáncer de mama y autoexamen». Revista Archivo Médico de Camagüey, Vol. 14, No 1, Camagüey, AMC. <http://scielo.sld.cu/scielo.php?script=sci_arttext\&pi$\mathrm{d}=$ S1025-02552010000100003\&lng=es\&nrm=iso $>$ [Consulta: 31-10-2015].

Organización Mundial de la Salud. 2015. Cáncer de mama: Prevención y control. Ginebra: WHO. <http://www.who.int/topics/cancer/breastcancer/es/> [Consulta: 3110-2015].

Yépez, Daniel et al. 2012. «Autoexploración mamaria: conocimiento y perspectiva en mujeres». Revista de Enfermería del Instituto Mexicano del Seguro Social, Vol. 20, Nº 2. Guanajuato: Instituto Mexicano del Seguro Social. 79-84.

Fecha de recepción: 15 de febrero 2016

Fecha de aceptación: 25 de mayo 2016

\section{Correspondencia}

Augusto Félix Olaza Maguiña

roke102030@hotmail.com 